\title{
Correlation of Anemia and Serum Transferrin in Diabetic Nephropathy
}

\author{
Dr. Naveen Kumar Sambu ${ }^{1 *}$, Dr. Durga Prasad Kedam², Dr. Havilah Polur ${ }^{3}$, Mr. Suman Putta ${ }^{4}$ \\ ${ }^{1}$ Assistant Professor, Departmet of Biochemistry, Santhiram Medical College \& General Hospital, NH 18, Kurnool Dist., Nandyala, 518001, India \\ ${ }^{2}$ Professor \& Head, Departmet of Biochemistry, Santhiram Medical College \& General Hospital, NH 18, Kurnool Dist., Nandyala, 518001, India \\ ${ }^{3}$ Professor, Departmet of Biochemistry, Santhiram Medical College \& General Hospital, NH 18, Kurnool Dist., Nandyala, 518001, India \\ ${ }^{4}$ Lecturer, Departmet of Biochemistry, Santhiram Medical College \& General Hospital, NH 18, Kurnool Dist., Nandyala, 518001, India
}

DOI: $10.36348 /$ sijb.2020.v03i01.002

| Received: 21.01.2020 | Accepted: 28.01.2020 | Published: 30.01 .2020

*Corresponding author: Naveen Kumar Sambu

\section{Abstract}

Background: End Stage Renal Disease is the common cuase of morbidity and mortality in patients suffering from diabetes and hypertension. Loss of transferrin and renal impairment in diabetic patients might lead to microcytic anaemia. Progression of nephropathy in these patients can be prevented by checking the hemoglobin levels. Objectives: This study is aimed at identifying the relationship of transferrin levels and anaemia in diabetic nephropathy. Methods: The study included 100 patients who were categorized as 20 normoalbuminuric, 40 microalbuminuric and 40 macroalbuminruic based on urine albumin levles. Serum Transferrin, Hemoglobin were measured in all the three groups. Results: The data obtained concludes that the levels of hemoglobin are significantly reduced in macroalbuminuric $(9.0 \pm 1.61)$ than mciroalbuminuric $(11.5 \pm 1.71)$ as compared to normoalbuminuric $(13.1 \pm 1.91)$ patients. Serum transferrin levels were significantly reduced in macroalbuminuria $(207 \pm 33.7)$ as compared to normoalbuminuria $(263 \pm 51.8)$, which in correlation to hemoglobin levels. Conclusion: Anaemia was often seen at an early stage in diabetic nephropathy than in patients with chronic kidney disease. It is, therefore, crucial to monitor anaemia to prevent the progression of renal disease in diabetic patients manifested as microalbuminuria. Plasma transferrin levels are decreased in macroalbuminuria though the synthesis is increased in diabetic nephropathy as the response doesn't compensate for the loss of transferrin in the urine. Further understanding of the mechanism and providing the therapy may improve patient outcomes.

Keywords: End Stage Renal Disease, Transferrin, Diabetic Nephropathy, Albuminuria.

Copyright @ 2020: This is an open-access article distributed under the terms of the Creative Commons Attribution license which permits unrestricted use, distribution, and reproduction in any medium for non-commercial use (NonCommercial, or CC-BY-NC) provided the original author and source are credited.

\section{INTRODUCTION}

Patients with diabetes suffer the implications of impaired renal function earlier in the course of their disease than do their non-diabetic counterparts [1-3]. In diabetic nephropathy, anaemia tends to be more severe than in non-diabetic renal disease and occur at an earlier stage of the illness [4]. However, because most patients with early diabetic nephropathy have little overt renal impairment, primary care physicians are often the firstline health care providers and may not be aware of the critical importance of screening for anaemia. Thus anaemia could typically be unrecognised or untreated [5].

Anaemia outlined as haemoglobin $<13 \mathrm{gm} / \mathrm{dl}$ in males and $<12 \mathrm{gm} / \mathrm{dl}$ in females [6,7]. Diabetes is the single commonest reason behind end-stage renal disease and thus, the foremost common reason behind nephritic anaemia [8]. The predominance of harm to the renal interstitium, systemic inflammation and autonomic nephropathy have all been suggested as contributing to anaemia in diabetic nephropathy [9]. Microalbuminuria develops after five years of diabetes whereas positive dipstick proteinuria develops 5 to 10 years after the onset of microalbuminuria and is associated with progressive loss of renal function [10]. Validating clues for nephropathy include enlarged kidneys and evidence of diabetic retinopathy. Anaemia is a common accompaniment of diabetes, particularly in patients with albuminuria or decreased renal function. Studies in patients with renal impairment suggest that deleterious effect begins with haemoglobin $<11 \mathrm{gm} / \mathrm{dl}$ meaning that $7 \%$ of patients with diabetes may benefit from intervention according to current guidelines [11]. Studies have shown the importance of iron stores in the development and progression of anaemia in patients with diabetes.

Transferrin is the principal plasma protein for the transport of iron; each molecule of transferrin has two binding sites for metal with totally different affinities at physiological $\mathrm{pH}$. Transferrin is a negative 
acute-phase protein with a molecular weight of eighty kilo daltons synthesised with in the liver [12]. Plasma levels of transferrin are regulated primarily by the availability of iron [13]. Tranferrinuria, particularly with impaired renal functions and proteinuria, also contribute to iron deficiency anaemia [14]. Very few studies have been done on transferrin levels in diabetes with and without nephropathy. The present study aims to elicit the relationship of transferrin levels and anaemia in diabetic nephropathy.

\section{MATERIALS AND METHODS}

The current study is a Cross-Sectional study conducted in our hospital from July 2018 to June 2019. The study had 100 patients who were categorised as the group I, 20 diabetics with normoalbuminuria $(<30$ $\mathrm{mg} /$ day), group II, 40 diabetics with microalbuminuria (30-300 mg/day) and group III, 40 diabetics with macroalbuminuria (>300 mg/day).

Inclusion Criteria Include: Patients with type 2 diabetes of age $>35$ years.

Exclusion Criteria Includes: Acute infections, Congestive Cardiac failure, Uncontrolled hypertension, other causes of iron deficiency anaemia and pregnancy.

The study was approved from the ethics committee of our Institute. Patients were provided with complete information, and written consent is obtained.

Diagnosis of diabetes is based on WHO criteria, i.e.; FBG > $126 \mathrm{mg} / \mathrm{dl}$ or postprandial glucose $>150 \mathrm{mg} / \mathrm{dl}$, similarly anemia being defined as hemoglobin $<13 \mathrm{gm} / \mathrm{dl}$ in males and $<12 \mathrm{gm} / \mathrm{dl}$ in females.

\section{Biochemical Methods}

- Glucose - GOD POD method

- Hemoglobin - Hematology Analyser

- HbA1c-Turbidometric method
- Creatinine - Enzymatic method

- Urine microalbumin - Turbidometric method

\section{Estimation of Serum Transferrin}

Serum Transferrin was determined by Immunoturbidometric method. Mixing of the sample with a precise antigen to a solution having corresponding anti-serum in a well-defined ratio, it is possible to have turbidity. Plotting on the calibration curve absorbance values and concentration for every single sample may determine the level of each sample.

\begin{tabular}{|l|l|l|l|}
\hline & Blank & Standard & Test \\
\hline Reagent & $500 \mu \mathrm{L}$ & $500 \mu \mathrm{L}$ & $500 \mu \mathrm{L}$ \\
\hline Sample & - & $6 \mu \mathrm{L}$ & - \\
\hline Calibrator & - & - & $6 \mu \mathrm{L}$ \\
\hline
\end{tabular}

Mix carefully and incubate at $37^{\circ} \mathrm{C}$ for 5 minutes and measure the absorbance of test and calibrator against blank and calculate the test.

\section{STATISTICAL METHODS}

All the values were calculated as the mean \pm standard deviation. P-value was calculated, and $<0.05$ is taken as significant. Student T-test is used. Licensed SPSS software is used for the analysis of the data.

\section{RESULTS}

Table-1 shows the correlation between normoalbuminuria ( $<30 \mathrm{mg} /$ day) and microalbuminuria (30-300mg/day) in type 2 diabetes. Urine albumin is significantly $(\mathrm{p}=0.0003)$ elevated in microalbuminuria as compared to normoalbuminuria. Haemoglobin levels were not decreased significantly in microalbuminuria patients as compared to normoalbuminuria. Even serum transferrin and serum creatinine as haemoglobin have no significant change. A significant elevation of $\mathrm{HbA}_{1 \mathrm{C}}$, an index of blood glucose in the past three months is observed in microalbuminuria when compared to normoalbuminuria.

Table-1: Comparison of Normoalbuminuria with Microalbuminuria.

\begin{tabular}{|l|l|l|l|}
\hline & Group I (<30 mg/day) & Group II $\mathbf{~ ( 3 0 - 3 0 0 ~} \mathbf{~ m g} / \mathbf{d a y})$ & p-Value \\
\hline Urine Albumin $(\mathbf{m g} / \mathbf{d a y})$ & $21 \pm 8.9$ & $144.2 \pm 88.0$ & 0.0003 \\
\hline Serum Transferrin $(\mathbf{m g} / \mathbf{d l})$ & $263 \pm 51.8$ & $302 \pm 63.8$ & 0.1520 \\
\hline Hemoglobin $(\mathbf{g m} / \mathbf{d l})$ & $13.1 \pm 1.91$ & $11.5 \pm 1.71$ & 0.064 \\
\hline HbA $_{\mathbf{1 C}}(\mathbf{\%})$ & $6.09 \pm 0.5$ & $7.5 \pm 0.7$ & 0.0001 \\
\hline Serum Creatinine (mg/dl) & $0.93 \pm 0.3$ & $1.0 \pm 0.27$ & 0.241 \\
\hline
\end{tabular}

Table-2: Comparison of Microalbuminuria with Macroalbuminuria.

\begin{tabular}{|l|l|l|l|}
\hline & Group II (30-300 mg/day) & Group III (>300 mg/day) & p-Value \\
\hline Urine Albumin (mg/day) & $144.2 \pm 88.0$ & $778.3 \pm 339.9$ & 0.0001 \\
\hline Serum Transferrin (mg/dl) & $302 \pm 63.8$ & $207 \pm 33.7$ & 0.0006 \\
\hline Hemoglobin $(\mathbf{g m} / \mathbf{d l})$ & $11.5 \pm 1.71$ & $9.0 \pm 1.61$ & 0.004 \\
\hline HbA $_{\mathbf{1 C}}(\boldsymbol{\%})$ & $7.5 \pm 0.7$ & $9.6 \pm 1.7$ & 0.002 \\
\hline Serum Creatinine (mg/dl) & $1.0 \pm 0.27$ & $1.5 \pm 0.24$ & 0.002 \\
\hline
\end{tabular}


Table-2 shows the comparison of microalbuminuria (30-300mg/day) to macroalbuminuria (>300mg/day) in type 2 diabetes. Urine albumin was significantly elevated and haemoglobin levels are reduced significantly in macroalbuminuria as compared to microalbuminuria. A significant reduction is observed in serum transferrin and elevation in $\mathrm{HbA}_{1 \mathrm{C}}$ in macroalbuminuria as compared to microalbuminuria. A little elevation of serum Creatinine is observed.

Table III compares the normoalbuminuria with microalbuminuria in type 2 diabetes. All the parameters show a significant variations in normoalbuminuria compared to macroalbuminuria with type 2 diabetes.

Table-3: Comparison of Normoalbuminuria with Macroalbuminuria.

\begin{tabular}{|l|l|l|l|}
\hline & Group I (<30 mg/day) & Group III ( >300 mg/day) & p-Value \\
\hline Urine Albumin (mg/day) & $21 \pm 8.9$ & $778.3 \pm 339.9$ & $<0.0001$ \\
\hline Serum Transferrin (mg/dl) & $263 \pm 51.8$ & $207 \pm 33.7$ & 0.0107 \\
\hline Hemoglobin (gm/dl) & $13.1 \pm 1.91$ & $9.0 \pm 1.61$ & $<0.0001$ \\
\hline HbA $_{\mathbf{1 C}}(\%)$ & $6.09 \pm 0.5$ & $9.6 \pm 1.7$ & $<0.0001$ \\
\hline Serum Creatinine (mg/d) & $0.93 \pm 0.3$ & $1.5 \pm 0.24$ & 0.0003 \\
\hline
\end{tabular}

\section{DISCUSSION}

In our present study, we observed a nonsignificant reduction of $\mathrm{Hb}$ in microalbuminuric patients as compared to normoalbuminuric with type 2 diabetes mellitus. A significant decrease of $\mathrm{Hb}$ is observed in macroalbuminuric as compared to microalbuminuric with type 2 diabetes, which is in agreement with Ji suk Han study [15]. Diabetic nephropathy is associated with anaemia - defined as $\mathrm{Hb}<13 \mathrm{~g} / \mathrm{dl}$ in men and $\mathrm{Hb}<12 \mathrm{~g} / \mathrm{dl}$ in women. Various factors are responsible for the reduction in $\mathrm{Hb}$ in macroalbuminuric patients with type 2 diabetes such as deficiency of erythropoietin, urinary loss of transferrin, chronic inflammation, nutritional deficiency of iron, folate [16]. Decreased production of erythropoietin is an essential cause of anaemia in type 2 diabetes because of early damage to the renal tubular interstitium and the presence of autonomic neuropathy [17]. Another factor is inflammation, leading to the production of interleukin-6, tumour necrotic factor-alpha, which blunt the effect of erythropoietin on bone marrow and inhibit the stimulation of erythroid precursors, inhibiting erythropoiesis. Hepcidin plays a crucial role in iron metabolism. It increases the ferritin thus increasing the ability to sequester iron intracellularly but decreases the levels of transferrin; this reduces the intravascular transport of iron causing an iron deficiency in chronic inflammatory diseases [18]. Serum transferrin levels were non-significantly elevated in microalbuminuria as compared to normoalbuminuria and in macroalbuminuria, the levels were decreased significantly as compared to microalbuminuria, which indicates initial elevation was to compensate the reduced levels of haemoglobin and later decrease of transferrin in macroalbuminuria, though there was no improvement in haemoglobin might be due to the urinary losses of transferrin [19]. No significant difference in serum creatinine was observed in microalbuminuria as compared to normoalbuminuric with type 2 diabetes and a little elevation is seen in macroalbuminuric patients when compared to microalbuminuria patients with type 2 diabetes, showing the less significance of serum Creatinine in the early detection of diabetic nephropathy as compared to urine microalbumin. The results are in concomitant with Pradeep Kumar D studies [20]. A significant alteration in $\mathrm{HbA1c}$ was observed in all the three groups of type 2 diabetes which shows poor glycemic control aggravates the kidney damage in type 2 diabetes and the cause for the inflammatory changes occurring in diabetic patients.

\section{CONCLUSION}

Micoralbumin is an early marker of the progression of diabetid nephropathy. Although microalbumin is a standard marker for early detection of diabetic nephropathy, it is not sufficiently accurate predictor of nephropathy due to certain limitations. Anaemia was often seen at an early stage in diabetic nephropathy than in patients with chronic kidney disease. It is, therefore, crucial to monitor anaemia to prevent the progression of renal disease in diabetic patients manifested as microalbuminuria. Plasma transferrin levels are decreased in macroalbuminuria though the synthesis is increased in diabetic nephropathy as the response doesn't compensate for the loss of transferrin in the urine. Further understanding of the mechanism and providing the therapy may improve patient outcomes.

\section{REFERENCES}

1. Bosman, D. R., Winkler, A. S., Marsden, J. T., Macdougall, I. C., \& Watkins, P. J. (2001). Anemia with erythropoietin deficiency occurs early in diabetic nephropathy. Diabetes care, 24(3), 495-499.

2. Dikow, R., Schwenger, V., Schömig, M., \& Ritz, E. (2002). How should we manage anaemia in patients with diabetes?. Nephrology Dialysis Transplantation, 17(suppl_1), 67-72.

3. Deray, G., Heurtier, A., Grimaldi, A., Laumary, V., \& Isnard, B. C. (2004). Anemia \& Diabetes. American Journal of Nephrology, 24, 522.

4. Craig, K. J., Williams, J. D., Riley, S. G., Smith, H., Owens, D. R., Worthing, D., ... \& Phillips, A. O. (2005). Anemia and diabetes in the absence of nephropathy. Diabetes care, 28(5), 1118-1123. 
5. Thomas, R., Kanso, A., \& Sedor, J. R. (2008). Chronic kidney disease and its complications. Primary care: Clinics in office practice, 35(2), 329-344.

6. Beutler, E., \& Waalen, J. (2006). The definition of anemia: what is the lower limit of normal of the blood hemoglobin concentration?. Blood, 107(5), 1747-1750.

7. Pasupula, D. K., \& Reddy, P. S. (2014). When is a South Indian really anemic?. Indian Journal of Clinical Biochemistry, 29(4), 479-484.

8. Hamid, N., \& Mahmoud, R. K. (2015). Diabetes mellitus and renal failure: Prevention \& Management. Journal of Research in Medical Sciences. 20(11), 1112.

9. Merlin, C. T., Reichard, J. M., David, P., \& George, J. (2003). Unrecognised anemia in patients with diabetes. 26(4), 1164.

10. Hesmatollah, S., \& Isa, R. (2013). Diabetic kidney disease: Review of the current knowledge. Journal of Renal injury prevention. 2(2), 73.

11. Sarita, B., Brij, M. M., Vinod, K. A., \& Pradeep, G. T. (2016). Management of anemia in patients with diabetic kidney disease: A consensus statement. Indian Journal of Endocrinology \& Metabolism. 20(2), 268.

12. Luck, A. N., \& Mason, A. B. (2012). Transferrinmediated cellular iron delivery. In Current topics in membranes (Vol. 69, pp. 3-35). Academic Press.

13. Wallace, D. F. (2016). The regulation of iron absorption and homeostasis. The Clinical Biochemist Reviews, 37(2), 51.

14. Brown, E. A., Sampson, B., Muller, B. R., \&
Curtis, J. R. (1984). Urinary iron loss in the nephrotic syndrome--an unusual cause of iron deficiency with a note on urinary copper losses. Postgraduate medical journal,60(700), 125-128.

15. Han, J. S., Lee, M. J., Park, K. S., Han, S. H., Yoo, T. H., Oh, K. H., ... \& Kim, Y. H. (2015). Albuminuria as a risk factor for anemia in chronic kidney disease: result from the KoreaN Cohort Study for Outcomes in Patients with Chronic Kidney Disease (KNOW-CKD). PloS one, 10(10), e013947.

16. Babitt, J. L., \& Lin, H. Y. (2012). Mechanisms of anemia in CKD. Journal of the American Society of Nephrology, 23(10), 1631-1634.

17. Bajaj, S., Makkar, B. M., Abichandani, V. K., Talwalkar, P. G., Saboo, B., Srikanta, S. S., ... \& Abraham, G. (2016). Management of anemia in patients with diabetic kidney disease: A consensus statement. Indian journal of endocrinology and metabolism, 20(2), 268.

18. Ganz, T., \& Nemeth, E. (2012). Hepcidin and iron homeostasis. Biochimica et Biophysica Acta (BBA)-Molecular Cell Research, 1823(9), 14341443.

19. Al-Rubeaan, K., Siddiqui, K., Al-Ghonaim, M. A., Youssef, A. M., Al-Sharqawi, A. H., \& AlNaqeb, D. (2017). Assessment of the diagnostic value of different biomarkers in relation to various stages of diabetic nephropathy in type 2 diabetic patients. Scientific reports, 7(1), 1-9.

20. Dabla, P. K. (2010). Renal function in diabetic nephropathy. World journal of diabetes, 1(2), 48. 